\title{
Dual function of the polyketide synthase A of the nematode-trapping fungus Duddingtonia flagrans in pigment biosynthesis and regulation of trap formation
}

\author{
XI YU ${ }^{12 *}$, XIAOdI HU ${ }^{2}$ AND REINHARD FISCHER ${ }^{2}$ \\ ${ }^{1}$ Shanghai Engineering Research Center of Hadal Science \\ and Technology, College of Marine Sciences, Shanghai \\ Ocean University, Shanghai, China (*correspondence: \\ xiyu@hotmail.com) \\ ${ }^{2}$ Department of Applied Microbiology, Karlsruhe Institute of \\ Technology, 76131 Karlsruhe, Germany \\ (huxiaodi369@163.com)
}

Nematode-trapping fungi (NTF) are a large and diverse group of fungi, which may switch from a saprotrophic to a predatory livestyle if nematodes are present. Secondary metabolites play important roles in the function and evolution of fungi, such as the nematicidal and antimicrobial activity in nematophagous fungi. Based on the genomic annotation of Duddingtonia flagrans we have got, the preliminary analysis revealed the presence of 29 putative SM-cluster, 4 PKS/PKS-NRPS hybrids and 3 NRPS genes, besides 2 terpenoid synthases. One of the PKS displays similarity to 6MSAS type polyketide synthases, which is negatively involved in trap formation in D. flagrans. The lack of pksA can lead to increased numbers of the traps. Importantly, when the pksA is deleted, it interferes the chemotaxis, suggesting that pksA affect the production of volatile compounds to attract C. elegans. The pigment of D. flagrans can be synthesized by two pathways, DHN-melanin pathway and LDOPA pathway. PksA mediates the biosynthesis of the pigment production and other secondary metabolites. Nematicidal activities of crude extracts and the virulence assay are also identified in the pksA mutant. It's believed that the exploration of related secondary metabolites genes is one of the key points to decipher the connection between the two species during the co-evolution of life and sheds light on the biological research in the deep sea biosphere. 